\title{
Resection of a Lower Clival Meningioma via Posterolateral Approach: Two-Dimensional Operative Video
}

\author{
Pinar E. Ocak ${ }^{1,2}$ Selcuk Yilmazlar ${ }^{2}$ \\ ${ }^{1}$ School of Medicine, Center for Neuroscience Research, Loma Linda \\ University, Loma Linda, California, United States \\ 2 Department of Neurosurgery, Uludag University School of Medicine, \\ Bursa, Turkey \\ J Neurol Surg B 2021;82(suppl S1):S31-S32.
}

Address for correspondence Pinar E. Ocak, MD, FEBNS, School of Medicine, Center for Neuroscience Research, Loma Linda University School of Medicine, Loma Linda, CA 92350, United States (e-mail: pocak@llu.edu).

\begin{abstract}
Keywords

- clival meningioma

- posterolateral approach

- craniovertebral junction

- vertebral artery

Objectives This study aimed to demonstrate resection of a craniovertebral junction (CVJ) meningioma via the posterolateral approach.

Design The study is designed with a two-dimensional operative video.

Setting This study is conducted at department of neurosurgery in a university hospital. Participants A 50-year-old woman who presented with lower cranial nerve findings due to a left-sided lower clival meningioma (- Fig. 1).

Main Outcome Measures Microsurgical resection of the meningioma and preservation of the neurovascular structures.

Results The patient was placed in park-bench position and a left-sided retrosigmoid suboccipital craniotomy, followed by $\mathrm{C} 1$ hemilaminectomy and unroofing the lip of the foramen magnum, was performed. The dural incision extended from the suboccipital region down to the posterior arch of C2 (-Fig. 2). The arachnoid overlying the tumor was incised, revealing the course of the cranial nerve (CN) XI on the dorsolateral aspect of the tumor. The left vertebral artery (VA) was encased by the tumor which was originating from the dura below the jugular foramen. The mass was resected in a piecemeal fashion eventually. At the end of the procedure, all relevant cranial nerves and adjacent vascular structures were intact. Postoperative magnetic resonance imaging (MRI) confirmed total resection and the patient was discharged home on postoperative day 3 safely.

Conclusions Microsurgical resection of the lesions of the CV] are challenging as this transition zone between the cranium and upper cervical spine has a complex anatomy. Since adequate exposure of the extradural and intradural segments of the VA can be obtained by the posterolateral approach, this approach can be preferred in cases with tumors anterior to the VA or when the artery is encased by the tumor.

The link to the video can be found at: https://youtu.be/d3u5Qrc-zIM.
\end{abstract}

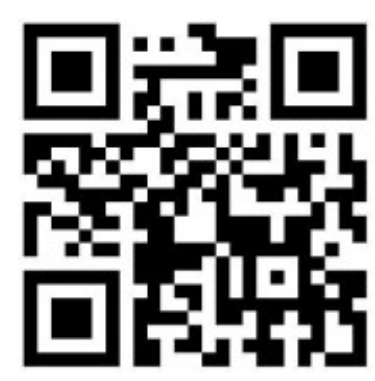

received

January 29, 2019

accepted

March 8, 2020

published online

December 29, 2020

www.thieme.com/skullbasevideos

www.thieme.com/jnlsbvideos

DOI https://doi.org/ 10.1055/s-0040-1714407. ISSN 2193-6331. (c) 2020. The Author(s).

This is an open access article published by Thieme under the terms of the Creative Commons Attribution-NonDerivative-NonCommercial-License, permitting copying and reproduction so long as the original work is given appropriate credit. Contents may not be used for commercial purposes, or adapted, remixed, transformed or built upon. (https://creativecommons.org/ licenses/by-nc-nd/4.0/)

Georg Thieme Verlag KG, Rüdigerstraße 14, 70469 Stuttgart, Germany 


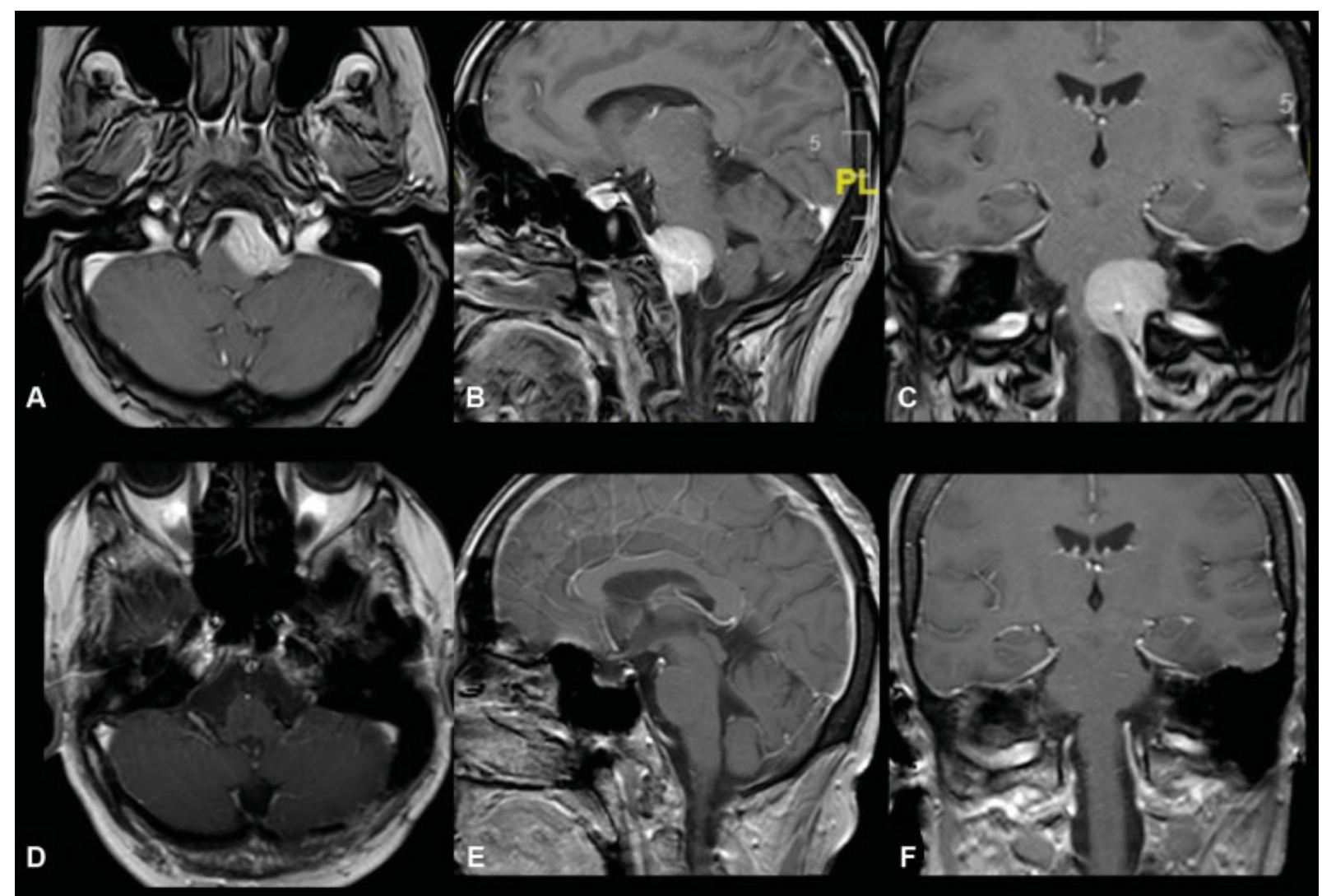

Fig. 1 Preoperative T1-weighted postcontrast axial (A), sagittal (B), and coronal (C) MR images of a 50-year-old female patient, demonstrating a diffusely enhancing mass lesion originating from the left lower clival region. (D-F) Postoperative MR images of the same patient, demonstrating gross total resection of the tumor. MR, magnetic resonance.

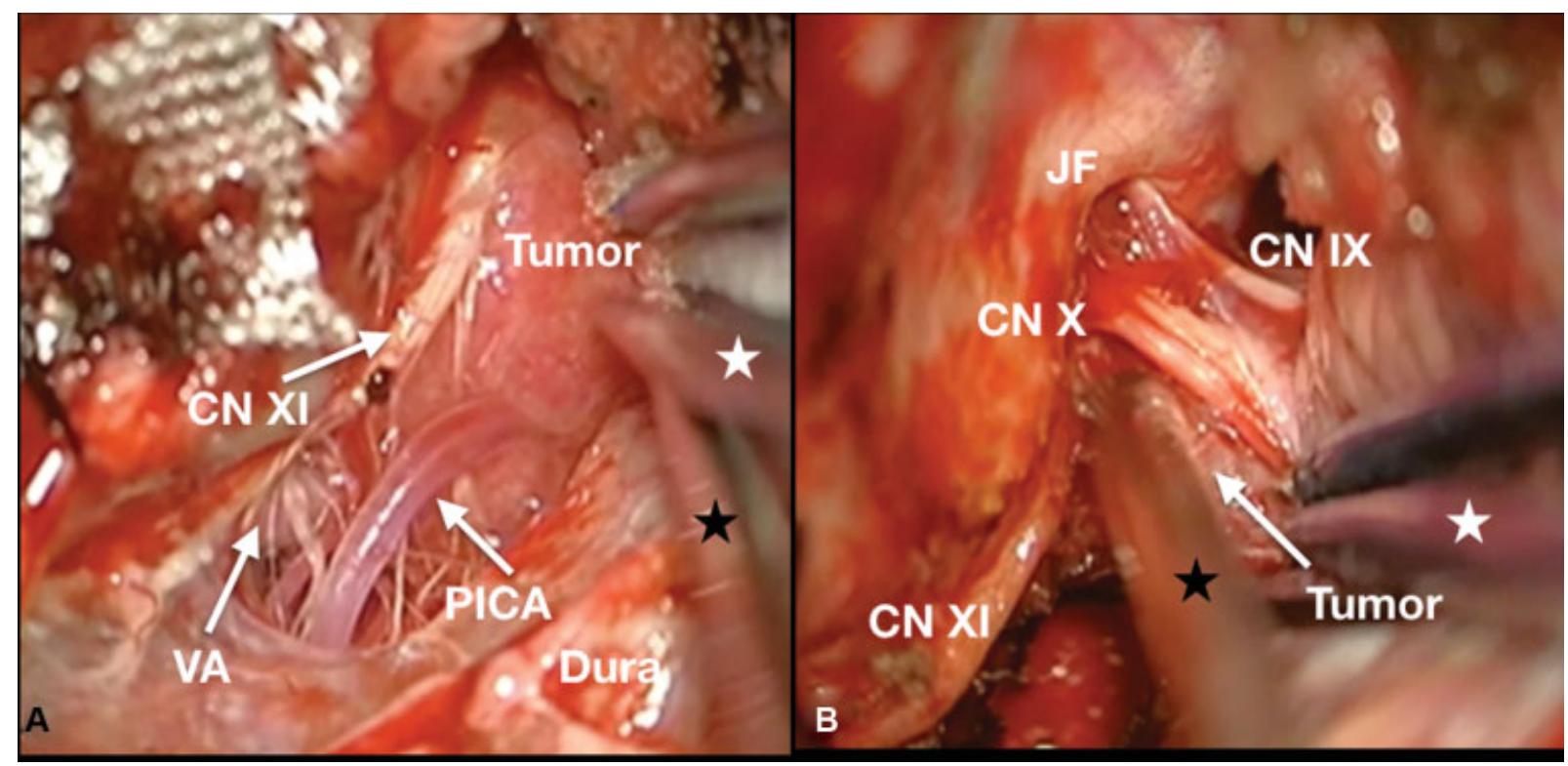

Fig. 2 Intraoperative microscopic images (A, B) demonstrating the close relation of the tumor with the adjacent neurovascular structures and (C, D) the location of the tumor anterior to the CN IX, X and XI (white stars: tip of the bipolar; black stars: tip of the suction). CN, cranial nerve; JF, jugular foramen; PICA, posterior inferior cerebellar artery; VA, vertebral artery.

Disclosure of Funding

None.
Conflict of Interest

None declared. 